\title{
GIBBS PHENOMENON FOR FUNCTIONS OF TWO VARIABLES $\left({ }^{1}\right)$
}

\author{
BY \\ FRED USTINA
}

1. Introductory remarks. Gibbs phenomenon, which concerns the nonuniform convergence of the partial sums of the Fourier series of a function in the neighborhood of a nonremovable discontinuity, was discovered by $\mathrm{H}$. Wilbraham [4] in 1848. It has since been studied very extensively in the case of functions of one variable. In the multi-dimensional case, however, very little appears to have been done. In fact, to our knowledge, there is only one published account of an investigation in the multi-dimensional case, that of Cheng [1] for two dimensions.

In the one-dimensional case, it is well known that to study the Gibbs phenomenon for the class of functions which are of bounded variation and which have no removable discontinuities, it is sufficient to study one such function. It is likely that the lack of an analogous result in the two-dimensional case has prevented its wider investigation here. When we go from the one- to the two-dimensional case, the problem immediately becomes much more complex because here the definition of bounded variation is no longer unique.

We investigate the class of functions which are of bounded variation in the sense of Hardy-Krause, and prove that to study the Gibbs phenomenon for this class of functions, it is sufficient to study it for one such function. We remark that this result generalizes the results obtained by Cheng, since he limited his investigations to functions which factorize into a product of two functions of bounded variation: $f(x, y)=f_{1}(x) \cdot f_{2}(y)$.

2. Preliminary remarks. As our starting point, we give a few of the basic definitions.

Definition 1. By a cell $\left[a_{2}, b_{2} ; a_{1}, b_{1}\right]$ in the real plane, we shall mean a rectangular point set determined by the vertices $\left(a_{1}, b_{1}\right),\left(a_{1}, b_{2}\right),\left(a_{2}, b_{1}\right)$ and $\left(a_{2}, b_{2}\right)$. As on the real line, a cell may be closed, open or halfopen.

Definition 2. If $f(x, y)$ is a real valued function of the variables $x$ and $y$, then

$$
f\left(a_{2}, b_{2} ; a_{1}, b_{1}\right)=f\left(a_{2}, b_{2}\right)-f\left(a_{2}, b_{1}\right)-f\left(a_{1}, b_{2}\right)+f\left(a_{1}, b_{1}\right) .
$$

Definition 3. Let $f(x, y)$ be defined on the cell $\left[a_{2}, b_{2} ; a_{1}, b_{1}\right], a_{1}<a_{2}$ and

Received by the editors November 4, 1966.

(1) The author is indebted to Professor Arthur E. Livingston, who suggested a thesis topic which led to this investigation. 
$b_{1}<b_{2}$. Let $\left\{x_{i}\right\}_{0}^{m}$ and $\left\{y_{i}\right\}_{0}^{n}$ be two sequences such that $a_{1}=x_{0}<x_{1}<\cdots<x_{m}=a_{2}$ and $b_{1}=y_{0}<y_{1}<\cdots<y_{n}=b_{2}$, and form the sum

$$
\sum_{i, j=1}^{m, n}\left|f\left(x_{i}, y_{j} ; x_{i-1}, y_{j-1}\right)\right| \text {. }
$$

If for all such partitions this sum is uniformly bounded, and $M$ is its least upper bound, and if for some fixed $\left(x^{\prime}, y^{\prime}\right) \in\left[a_{2}, b_{2} ; a_{1}, b_{1}\right], f\left(x, y^{\prime}\right)$, resp. $f\left(x^{\prime}, y\right)$, is a function of bounded variation as a function of $x$, resp. of $y$, then $f(x, y)$ is said to be of bounded variation in the sense of Hardy-Krause, and $M$ is its total variation.

REMARKS. For the various ways of defining the variation of a function of two variables, the reader is referred to Clarkson and Adams [2].

Definition 4. Let $\left\{\varepsilon_{n}\right\}$ be a sequence of positive numbers such that $\lim \varepsilon_{n}=0$, and let $\left\{p_{n}\right\}$ be a sequence of positive integers such that $\lim p_{n}=\infty$, the limit in both cases being taken as $n \rightarrow \infty$. Let $\left\{s_{m, n}(x, y)\right\}$ be a sequence of real valued functions of the variables $x$ and $y$. Then

$$
\begin{aligned}
& \quad \lim _{m, n \rightarrow \infty:(x, y) \rightarrow\left(x_{0}, y_{0}\right)} s_{m, n}(x, y) \\
& \quad=\lim _{k \rightarrow \infty} \sup \left\{s_{m, n}(x, y): m, n>p_{k} \text { and } 0<\left|x-x_{0}\right|+\left|y-y_{0}\right|<\varepsilon_{k}\right\} .
\end{aligned}
$$

The lim inf as $m, n \rightarrow \infty$ and as $(x, y) \rightarrow\left(x_{0}, y_{0}\right)$ is similarly defined.

Definition 5. If the sequence $\left\{s_{m, n}(x, y)\right\}$ converges pointwise to a limit function $f(x, y)$ in the region $0<\left|x-x_{0}\right|+\left|y-y_{0}\right|<\varepsilon$, then $f(x, y)$ is said to exhibit the Gibbs phenomenon at $\left(x_{0}, y_{0}\right)$ if one or both of the following inequalities hold:

$$
\begin{aligned}
& \lim \sup s_{m, n}(x, y)>\lim \sup f(x, y), \\
& \lim \inf s_{m, n}(x, y)<\lim \inf f(x, y),
\end{aligned}
$$

where both the lim sup and the lim inf are taken as $m, n \rightarrow \infty$ and $(x, y) \rightarrow\left(x_{0}, y_{0}\right)$.

Definition 6. Let $f(x, y)$ exhibit the Gibbs phenomenon at the point $\left(x_{0}, y_{0}\right)$. By the Gibbs ratio for $f(x, y)$ at the point $\left(x_{0}, y_{0}\right)$, we shall mean the real, positive number defined by

$$
\frac{\lim \sup s_{m, n}(x, y)-\lim \inf s_{m, n}(x, y)}{\lim \sup f(x, y)-\lim \inf f(x, y)}
$$

where again lim sup and lim inf are taken as $m, n \rightarrow \infty$ and as $(x, y) \rightarrow\left(x_{0}, y_{0}\right)$.

REMARKS. It would appear that the above definition of Gibbs ratio fails at those points where the limit of $f(x, y)$ exists. However, we will deal only with the case where $\left\{s_{m, n}(x, y)\right\}$ is the sequence of partial sums of the Fourier series of a function which is of bounded variation in the sense of Hardy-Krause. By Theorem 1 of [3], it follows that $f(x, y)$ cannot present the Gibbs phenomenon at such a point. Hence such points are excluded from our discussion.

3. Gibbs phenomenon for functions of two variables. We are now ready to state our main result. 
THEOREM. Let $f(x, y)$ be a $2 \pi$-periodic function having no removable discontinuities, and let $f(x, y)$ be of bounded variation in the sense of Hardy-Krause. The Gibbs phenomenon for the partial sums of the Fourier series of $f(x, y)$ at the point $\left(x_{0}, y_{0}\right)$ $=(0,0)$ is the same as the Gibbs phenomenon for the partial sums of the Fourier series of the function

$$
\psi(f ; x, y)=\left(c / \pi^{2}\right) \phi(x) \cdot \phi(y)+g_{1}(0) \phi(x)+g_{2}(0) \phi(y)
$$

where

$$
\begin{array}{rlrl}
\phi(t) & =0, & t=0, \\
& =\frac{1}{2}(\pi-t), & 0<t<2 \pi, \\
& =\phi(t+2 k \pi), \quad k= \pm 1, \pm 2, \pm 3, \ldots, \\
c & =f\left(0^{+}, 0^{+} ; 0^{-}, 0^{-}\right), \\
g_{1}(y) & =(1 / \pi)\left\{f\left(0^{+}, y\right)-f\left(0^{-}, y\right)\right\}-(c / 2 \pi) \operatorname{sgn} y, \\
g_{2}(x) & =(1 / \pi)\left\{f\left(x, 0^{+}\right)-f\left(x, 0^{-}\right)\right\}-(c / 2 \pi) \operatorname{sgn} x .
\end{array}
$$

COROLlaRY. To study the Gibbs phenomenon for an arbitrary function of bounded variation in the sense of Hardy-Krause, it is sufficient to study it for the functions $\phi(x), \phi(y)$ and $\phi(x) \cdot \phi(y)$.

REMARKS. It is clear that the only hypothesis which restricts the generality of the result is that which requires the function to be of bounded variation in the sense of Hardy-Krause.

Note that if the difference functions $f\left(x, 0^{+}\right)-f\left(x, 0^{-}\right)$and $f\left(0^{+}, y\right)-f\left(0^{-}, y\right)$ are continuous at the origin, then $c=0$ and $\psi(f ; x, y)=g_{1}(0) \phi(x)+g_{2}(0) \phi(y)$. In addition, if $f(x, y)$ is continuous along one of the two axes, say the $x$-axis, with the exception of the origin, then $g_{2}(x) \equiv 0$, and $\psi(f ; x, y)=g_{1}(0) \phi(x)$.

In both these cases, the Gibbs ratio is easily shown to be

$$
\frac{2}{\pi} \int_{0}^{\pi} \frac{\sin t}{t} d t
$$

In general, however, the expression for the Gibbs ratio does not reduce to this simple form.

In general, for ordinary convergence, the "overshoot" is not of the same magnitude as the "undershoot." This follows the observation that regardless of the signs of $g_{1}(0), g_{2}(0)$ and $c$, there is always one quadrant in which the terms $c \phi(x) \cdot \phi(y), g_{1}(0) \phi(x)$ and $g_{2}(0) \phi(y)$ are either all positive or all negative for a sufficiently small neighborhood of the origin. But there are never two quadrants, one in which they are all positive, and another in which they are all negative, unless $c=0$.

4. Proof of the Theorem. We assume that $f(x, y)$ is normalized. The discontinuities of $f(x, y)$ are then of a nonremovable nature, and occur only along lines which are parallel to the coordinate axes. Clearly we may assume that $f(x, y)$ 
is discontinuous along the coordinate axes, and in fact we may assume that the only discontinuities of $f(x, y)$ in the cell $(\pi, \pi ;-\pi,-\pi)$ are along the coordinate axes. For otherwise we could choose a sufficiently small neighborhood of the origin in which this is true, and limit our argument to that neighborhood.

By the corollary to Theorem 1 of [3], to prove the theorem it is sufficient to prove that $f(x, y)-\psi(f ; x, y)$ is continuous at $(0,0)$. From this it will follow that the Fourier series of $f(x, y)-\psi(f ; x, y)$ cannot show the Gibbs phenomenon there, and so the Gibbs phenomenon for $f(x, y)$ must be the same as for the function $\psi(f ; x, y)$. We do this in three stages. Let

$$
\pi g_{1}^{\prime}(y)=f\left(0^{+}, y\right)-f\left(0^{-}, y\right), \quad \pi g_{2}^{\prime}(x)=f\left(x, 0^{+}\right)-f\left(x, 0^{-}\right),
$$

and observe that $g_{1}^{\prime}(y)$ and $g_{2}^{\prime}(x)$ are both continuous or both discontinuous at the origin, for

$$
\begin{aligned}
\pi g_{1}^{\prime}\left(0^{+}\right)-\pi g_{1}^{\prime}\left(0^{-}\right) & =f\left(0^{+}, 0^{+}\right)-f\left(0^{-}, 0^{+}\right)-f\left(0^{+}, 0^{-}\right)+f\left(0^{-}, 0^{-}\right) \\
& =\pi g_{2}^{\prime}\left(0^{+}\right)-\pi g_{2}^{\prime}\left(0^{-}\right) .
\end{aligned}
$$

Next, let

$$
\begin{aligned}
\alpha(t) & =\pi / 2, & & 0<t<\pi, \\
& =0, & t & =0, \pi, \\
& =-\pi / 2, & -\pi & <t<0, \\
& =\alpha(t+2 k \pi), & k & = \pm 1, \pm 2, \pm 3, \ldots,
\end{aligned}
$$

and observe that

$$
h_{1}(x, y)=f(x, y)-\alpha(y) g_{2}^{\prime}(x)
$$

is continuous except along the $y$-axis.

Next, we consider the difference function $g\left(h_{1} ; y\right)$, where

$$
\pi g\left(h_{1} ; y\right)=h_{1}\left(0^{+}, y\right)-h_{1}\left(0^{-}, y\right) \text {, }
$$

and write

$$
h(x, y)=h_{1}(x, y)-\alpha(x) g\left(h_{1} ; y\right),
$$

and note that $h(x, y)$ is continuous in the cell $(\pi, \pi ;-\pi,-\pi)$. In particular, $h(x, y)$ is continuous at the origin.

Next, we decompose $g_{1}^{\prime}(y)$ and $g_{2}^{\prime}(x)$ into the continuous and discontinuous components:

$$
g_{1}^{\prime}(y)=g_{1}(y)+\alpha(y) c / \pi^{2}, \quad g_{2}^{\prime}(x)=g_{2}(x)+\alpha(x) c / \pi^{2} .
$$

Then by (1), (2), (3) and (4),

$$
\begin{aligned}
h(x, y)= & f(x, y)-\alpha(y) g_{2}^{\prime}(x)-(\alpha(x) / \pi) \\
& \cdot\left\{f\left(0^{+}, y\right)-\alpha(y) g_{2}^{\prime}\left(0^{+}\right)-f\left(0^{-}, y\right)+\alpha(y) g_{2}^{\prime}\left(0^{-}\right)\right\} \\
= & f(x, y)-\alpha(y) g_{2}(x)-\alpha(y) \cdot \alpha(x) c / \pi^{2}-\alpha(x) g_{1}(y)-\alpha(x) \alpha(y) c / \pi^{2} \\
& +\alpha(x) \alpha(y) \cdot(1 / \pi)\left\{g_{2}^{\prime}\left(0^{+}\right)-g_{2}^{\prime}\left(0^{-}\right)\right\} \\
= & f(x, y)-\alpha(y) g_{2}(x)-\alpha(x) g_{1}(y)-\alpha(x) \cdot \alpha(y) c / \pi^{2} .
\end{aligned}
$$


Thus along the lines of discontinuity, the Gibbs phenomenon for $f(x, y)$ is the same as the Gibbs phenomenon for the function

$$
\psi_{1}(f ; x, y)=\left(c / \pi^{2}\right) \alpha(x) \alpha(y)+\alpha(y) g_{2}(x)+\alpha(x) g_{1}(y) .
$$

Now let $f_{1}(x, y)$ be a function such that for oblique approach to the origin,

$$
f_{1}\left(0^{ \pm}, 0^{ \pm}\right)=f\left(0^{ \pm}, 0^{ \pm}\right)
$$

and such that for all $x$ and $y$ in the cell $(\pi, \pi ;-\pi,-\pi)$,

$$
f_{1}\left(0^{+}, y\right)-f_{1}\left(0^{-}, y\right)=g_{1}(0), \quad f_{1}\left(x, 0^{+}\right)-f_{1}\left(x, 0^{-}\right)=g_{2}(0) .
$$

That is, the continuous components of the difference functions of $f_{1}(x, y)$ are constant. It is clear that $f_{1}\left(0^{+}, 0^{+} ; 0^{-}, 0^{-}\right)=f\left(0^{+}, 0^{+} ; 0^{-}, 0^{-}\right)=c$. Then, writing

$$
\psi_{2}(f ; x, y)=\psi_{1}\left(f_{1} ; x, y\right)=\left(c / \pi^{2}\right) \alpha(x) \alpha(y)+\alpha(y) g_{2}(0)+\alpha(x) g_{1}(0),
$$

and so

$$
\psi_{1}(f ; x, y)-\psi_{2}(f ; x, y)=\alpha(y)\left\{g_{2}(x)-g_{2}(0)\right\}+\alpha(x)\left\{g_{1}(y)-g_{1}(0)\right\} .
$$

This function is continuous at the origin, this continuity being implied by the continuity of the functions $g_{2}(x)$ and $g_{1}(y)$ there. Hence the Gibbs phenomenon for $\psi_{2}(f ; x, y)$ at the origin must be the same as the Gibbs phenomenon for $\psi_{1}(f ; x, y)$, and so the same as for the function $f(x, y)$ there.

Finally, we define $\phi(t)$ as follows:

$$
\begin{aligned}
\phi(t) & =\frac{1}{2}(\pi-t), & & 0<t<2 \pi, \\
& =0, & & t=0, \\
& =\phi(t+2 k \pi), & & k= \pm 1, \pm 2, \pm 3, \ldots .
\end{aligned}
$$

Let

$$
\psi(f ; x, y)=\left(c / \pi^{2}\right) \phi(x) \phi(y)+g_{1}(0) \phi(x)+g_{2}(0) \phi(y),
$$

and form the difference function

$$
\begin{aligned}
\psi(f ; x, y)-\psi_{2}(f ; x, y)= & g_{2}(0)\{\phi(y)-\alpha(y)\} \\
& +g_{1}(0)\{\phi(x)-\alpha(x)\}+\left(c / \pi^{2}\right)\{\phi(x) \phi(y)-\alpha(x) \alpha(y)\} .
\end{aligned}
$$

This difference function is again continuous at the origin, and so the Gibbs phenomenon for $\psi(f ; x, y)$ there must be the same as the Gibbs phenomenon for $f(x, y)$.

We conclude the proof of the theorem by noting that

$$
\begin{aligned}
& g_{1}(y)=g_{1}^{\prime}(y)-\alpha(y)\left(c / \pi^{2}\right)=(1 / \pi)\left\{f\left(0^{+}, y\right)-f\left(0^{-}, y\right)\right\}-(c / 2 \pi) \operatorname{sgn} y \\
& g_{2}(x)=g_{2}^{\prime}(x)-\alpha(x)\left(c / \pi^{2}\right)=(1 / \pi)\left\{f\left(x, 0^{+}\right)+f\left(x, 0^{-}\right)\right\}-(c / 2 \pi) \operatorname{sgn} x .
\end{aligned}
$$

5. Remarks. We note that the extension of the theorem is not possible for the class of functions which are of bounded variation in the sense of Vitali or in the sense of Fréchet. For let $f_{1}(x) \equiv 1$ and let $f_{2}(y)$ be a bounded, continuous function 
of period $2 \pi$ which is of unbounded variation, and whose Fourier series diverges at a point $y_{0}$. Then $f(x, y) \equiv f_{1}(x) f_{2}(y)$ is of bounded variation in the sense of Vitali and in the sense of Fréchet in the period square $[2 \pi, 2 \pi ; 0,0]$, and its Fourier series diverges at every point $\left(x, y_{0}\right)$, which is also a point of continuity of $f(x, y)$. As we have seen, the proof of the theorem depends on the result that the Fourier series of a function of bounded variation in the sense of Hardy-Krause converges to the value of the function at every point of continuity of the function, this convergence being uniform in some neighborhood of such a point.

Also, it appears unlikely that the theorem can be extended to the classes of functions. which are of bounded variation in the sense of Arzela, Pierpont or Tonelli, without some additional restrictions on these classes.

\section{REFERENCES}

1. M. Cheng, The Gibbs phenomenon and Bochner's summation method. II, Duke Math. J. 17 (1950), 477-490.

2. J. A. Clarkson and C. R. Adams, On definitions of bounded variation for functions of two variables, Trans. Amer. Math. Soc. 35 (1933), 824-854.

3. F. Ustina, Convergence of the Hausdorff means of double Fourier series, (to appear).

4. H. Wilbraham, On a certain periodic function, Cambridge and Dublin Math. J. 3 (1848), 198-201.

5. A. Zygmund, Trigonometric series, Vol. I, Cambridge Univ. Press, New York, 1959.

UnIVERSity OF Alberta,

Edmonton, Alberta, Canada 